\title{
Dynamic Assessment of Figurative Language of Children in the Autistic Spectrum: The Relation to Some Cognitive and Language Aspects
}

\author{
David Tzuriel \\ Tamar Groman \\ Bar-Ilan University, Israel
}

\begin{abstract}
The objectives of this study were to examine (a) differences in figurative language, analogical reasoning, executive functions (EF), theory of mind (ToM), and local/ central coherence (LCC) of children with high-functioning autism (HFA; $n=32$ ) and typically developing (TD; $n=32$ ) children; (b) improvement of figurative language using dynamic assessment; and (c) prediction of proverbial understanding by the cognitive variables. A sample of 5- to 11-year-old children with HFA was pair matched with a group of TD children on age, gender, vocabulary, and socioeconomic status (SES). Participants were administered tests of proverbial understanding, metaphorical construction, analogies, language ability, EF, LCC, and ToM. TD children scored higher than children with HFA on all tests. In the HFA group, proverbial understanding was predicted by LCC and verbal ability and in the TD group by metaphorical construction and EF. These findings refute the argument that figurative language among HFA is a function of only verbal ability.
\end{abstract}

Keywords: figurative language; proverbial understanding; metaphorical construction; high-functioning autism (HFA); dynamic assessment; local/central coherence

$\mathrm{P}$ ragmatic language draws on many different knowledge bases and interacting cognitive systems (Martin \& McDonald, 2003; Rundblad \& Annaz, 2010; Whyte, Nelson, \& Kahn, 2011). Similarly, pragmatic deficits are likely to reflect a heterogeneous range of underlying functional deficits or abnormalities, as reflected by the large number of disorders which are characterized by pragmatic disturbance (Eales, 1993). One of the heated debates is to what degree difficulties in pragmatic understanding characterizing children in the autistic spectrum reflects unique autistic functioning or language difficulties. Several authors relate to pragmatic language difficulties as uniquely characterizing children in the autistic spectrum (e.g., Happé, 1993), whereas others argue that they reflect any child with low comprehension 
abilities (e.g., Gernsbacher \& Pripas-Kapit, 2012). Norbury (2004) who carried out a study on children's understanding of idioms concludes that comprehension ability per se is "the most important determinant of success" in comprehending idioms (p. 1190). Children with a disability in language comprehension, regardless of autistic status, have more difficulty understanding idioms than do children, with autism or not, without a language disability. Giora, Gazal, Goldstein, Fein, and Stringaris (2012) referring to Norbury's (2005a) study conclude that "only those autistic individuals with concomitant language disability have difficulty understanding potentially ambiguous terms" (p. 23). Thus, individuals with autism are not different from nonautistic peers. For both groups, the ability to understand ambiguous terms is determined by their comprehension skill, not their autistic traits.

In this study, an attempt was made to explore the contribution of language ability to figurative language as well as the differences between children with high-functioning autism (HFA) and typically developing (TD) children on two main modes of figurative language: proverbial understanding and metaphorical construction. We focus on these modes because they are considered as a major aspect of pragmatic language (e.g., Honeck, 1997; Nippold, 1998; Nippold, Uhden, \& Schwarz, 1997; Piaget, 1926). A novel aspect of this study is assessment of figurative language using a dynamic assessment (DA) approach. We expected DA to reveal higher performance than a standardized approach. Another focus of this study is the prediction of proverbial understanding by metaphorical construction, analogical reasoning, local/ central coherence (LCC), executive functions (EF), theory of mind (ToM), and verbal ability.

Figurative language of children in the autistic spectrum was found by many researchers to be deficient as compared with TD children (e.g., Dennis, Lazenby, \& Lockyer, 2001; Gold, Faust, \& Goldstein, 2010; Kerbel \& Grunwell, 1998; Le Sourn-Bissaoui, Caillies, Gierski, \& Motte, 2011; MacKay \& Shaw, 2004; Melogno, Pinto, \& Levi, 2012; Norbury, 2005a; Rapin \& Dunn, 2003; Rundblad \& Annaz, 2010; Worth \& Reynolds, 2008; Whyte et al., 2011). However, it is worth to mention that some researchers reported an appropriate metaphorical understanding of children with autism, although lower than TD children, and that individuals with autism performed at greater than chance levels on metaphors (Kasirer, \& Mashal, 2014; Olofson et al., 2014).

Three central theories of autism bring to light the relationships between autism and social-linguistic deficiencies: the weak central coherence theory (WCCT), ToM, and EF. According to the WCCT, individuals in the autistic spectrum show a tendency to local, detailed, and fragmented processing of information as compared with TD individuals who have a tendency to global processing of information. Frith and Happé (1994) suggest that autism is characterized by a weak or absent motivation to searching for a general coherence. Individuals with autism process things in a detail-focused or piecemeal way, processing the constituent parts, rather than the global whole. These tendencies delay individuals in the autistic spectrum to use higher meaning produced by the context as well as to understand figurative language. The research is replete with findings showing inefficient integration of linking between ambiguous sentences with previous context in individuals in the autistic spectrum. An extensive body of research related to gist-based processing demonstrates that individuals with autism do not use the resulting gist representations to generalize information to related situations, organize associated knowledge, and guide behavior as spontaneously or effectively as TD individuals (e.g., Bennetto, Pennington, \& Rogers, 1996; Boucher \& Bowler, 2008; Fein et al., 1996; Jolliffe \& Baron-Cohen, 1999; Miller, Odegard, \& Allen, 2014; Minshew \& Goldstein, 2001; Tager-Flusberg, 1991). However, they are able to use gist when explicitly 
directed or when task demands are low and encourage reliance on gist (Bennetto et al., 1996; Bigham, Boucher, Mayes, \& Anns, 2010; Minshew \& Goldstein, 2001; Williams, Goldstein, \& Minshew, 2006). Miller et al. (2014) claim that the typical developmental shift toward a gist-based processing bias is delayed or reduced in individuals with autism, but the ability to encode global and local gist is largely intact. This approach corresponds to the DA approach (see the following text).

Two major factors studied in relation to autism are ToM and EF. ToM is defined as the ability to infer to others' minds a range of mental states (beliefs, desires, intentions, imagination, emotions, etc.) that cause different behaviors (Wellman \& Liu, 2004). ToM has been related to the ability to process mental expressions for understanding, prediction, and judgment of behaviors (Le Sourn-Bissaoui et al., 2011) and is considered as necessary for participating in an effective communication (Martin \& McDonald, 2004). It has also a great impact on understanding and predicting of social behavior in the future, on relating to motivational sources of others, and understanding of pragmatic aspects of language (Baron-Cohen, 1995; Happé, 1993). EF is characterized by an ability to maintain an appropriate problem-solving set for attainment of a future goal. It includes behaviors such as planning, impulse control, inhibition, set maintenance, organized search, and flexibility of thought and action (Martin \& McDonald, 2003; Ozonoff, Pennington, \& Rogers, 1991). According to EF theory, there is a supermechanism that manages reactions to problems requiring planning and implementation of problem solving, focusing and splitting attention, and thinking and behavior flexibility that impact on coping with daily situations and academic abilities (Bauminger-Zviely, 2013; Jurado \& Rosselli, 2007; Ozonoff et al., 1991). Mashal and Kasirer (2011) found a relation between EF skills and metaphor understanding in a group of 12- to 15-year-old children with autism. The core of the difficulty has been attributed to overused metaphors. These metaphors are encoded in mental memory, and to understand them, an early comprehensive knowledge is required.

Another way to understand EF of children with autism is apparent in the cognitive complexity and control theory, according to which, language plays an important role in the conscious control over an action; natural language is held to be a medium through which higher order rules are formulated (Zelazo, 2004). In both ToM and EF theories, the use of rules that demand higher level thinking is emphasized. EF are also required for the transfer from local processing to global processing and therefore are adequate with WCCT (Happé \& Frith, 2006). According to Pellicano $(2007,2010)$, EF appears to be a significant predictive factor for ToM abilities in intellectually able preschool children with autism. Pellicano examined the longitudinal relationships between ToM, EF, and central coherence at 4-7 years and then 3 years later at age 7-10 years. One of the most important findings arisen from this study was that individual differences in EF at the first age period predicted the change in children's ToM skills at the second age period, over and above the variance that was accounted for by age, verbal and nonverbal abilities, and children's initial ToM. Furthermore, no links emerged between children's early ToM skills and their later EF abilities. The link between ToM and EF in preschool children with autism is also widely discussed in Kimhi, Shoam-Kugelmas, Agam Ben-Artzi, Ben-Moshe, and Bauminger-Zviely (2014), focusing on EF cognitive shifting and planning and ToM prediction and explanation abilities. In their research on preschoolers with autism spectrum disorder (ASD) and TD preschoolers, they found that in both groups, planning and cognitive shifting as well as verbal IQ contributed significantly in predicting of ToM abilities. Findings also showed that EF and ToM scores were significantly correlated 
in both groups. In sum, children with autism fail EF and ToM tests as a result of the tasks' executive or rule-use requirements (Zelazo, Jacques, Burack, \& Frye, 2002).

\section{THE DYNAMIC ASSESSMENT APPROACH}

DA refers usually to an assessment, by an active teaching process, of an individual's perception, learning, thinking, and problem solving. The process is aimed at modifying an individual's cognitive functioning using mediation strategies (e.g., scaffolding, rule teaching) and observing subsequent changes in learning and problem-solving patterns within the testing situation (Feuerstein, Rand, \& Hoffman, 1979; Tzuriel, 2001, 2012). DA has been motivated by the inadequacy of static tests to provide accurate information about the individual's learning ability, specific deficient functions, and specific learning and change processes (Haywood \& Lidz, 2007; Haywood \& Tzuriel, 1992, 2002; Lidz \& Elliott, 2000; Sternberg \& Grigorenko, 2002; Tzuriel, 2001, 2012). In this study, we used a DA approach with three of the main variables: proverbial understanding, metaphorical construction, and analogical reasoning (see "Measures" section). The DA approach is especially important with children demonstrating cognitive difficulties because it can reflect more accurately their learning potential. Research findings have shown that children with disabilities demonstrate higher improvements in cognitive tasks following a teaching phase within DA than those shown by TD children (e.g., Tzuriel, 2001). Previous findings have also shown that children in the autistic spectrum demonstrate an adequate level of cognitive modifiability (Haywood, 1995), and improvement in understanding metaphors (Persicke, Tarbox, Ranick, \& St. Clair, 2012), false belief of others (Ozonoff \& Miller, 1995), and in communicating in efficient ways in social situations (Owens, Granader, Humphrey, \& Baron-Cohen, 2008). In this study, we applied a DA approach with a group of children with HFA and TD children. We expected to find lower scores on all variables but higher improvements in the HFA than in the TD group on proverbial understanding, metaphorical construction, and analogical reasoning tests. We also expected that language ability will be central in predicting figurative language, especially in the HFA group together with variables of LCC, ToM, and EF.

\section{METHOD}

\section{Sample}

The sample was composed of a group of HFA children $(n=32)$, who were pair matched to a group of TD children $(n=32)$ on age, gender, vocabulary subtest (Wechsler Intelligence Scale for Children-Sixth Edition [WISC-VI]) and socioeconomic status (SES) living area. The mean age of the HFA and TD groups was 112 months $(S D=18.15)$ and 112 months $(S D=17.35)$, respectively. Each group included 24 boys and 8 girls. The participants were selected from schools in medium-high SES areas in the center of Israel. The participants came from two main sources: the school system and the Israeli Society for Autistic Children (ALUT). We contacted the children's parents through their school teachers and/or caregivers after obtaining permission for research from the Israeli Ministry of Education and University Ethics Committee. After receiving a written parental consent for participation, we advised the parents and the teachers about the nature of the research. All children with HFA were previously diagnosed by licensed psychologists based on the Diagnostic and Statistical Manual of Mental Disorders (4th ed.; American Psychiatric Association, 2000). Three children were 
diagnosed with Asperger's syndrome. Basing on the perception of Asperger's syndrome as a subgroup of autism (e.g., Rajendran \& Mitchell, 2007), we combined these two diagnostic subgroups into a single group of HFA. All children with HFA were previously diagnosed as having normal or above normal IQ based on the Wechsler Intelligence Scale for ChildrenFourth Edition (WISC-IV; Wechsler, 1950). The WISC-IV Vocabulary subtest administered to all participants with HFA showed a standard mean score of $10.27(S D=4.02)$.

\section{Measures}

Children's Proverbial Understanding Test. The Children's Proverbial Understanding Test (CPUT) is a DA measure (Tzuriel \& Valdman, 2009) for young children. The test construction was carried out in seven stages: (1) Sixty metaphorical proverbs were chosen from popular children's books. (2) The proverbs were scrutinized and short listed to 19 after consulting with preschoolers experts. (3) A suitable story was composed to each picture accompanied with an illustration that suits its figurative meaning. The need for an illustration is based on studies showing that children are more likely to produce a figurative meaning for an idiom, metaphor, or a proverb when they are presented in a context with a figurative meaning, rather than presented in isolation or a literal context (e.g., Levorato \& Cacciari, 1992). (4) A pilot study was carried out on 10 kindergarten children to examine how suitable are the proverbs for young children. Children were asked questions related to understanding of the proverb and its context and provide an example of the proverb in another context than the original situation. Thirteen proverbs were then chosen based on qualitative analysis of children's responses; 2 proverbs were chosen as examples and 11 as test items. (5) The proverbs have been worded with light syntactic changes to make them more understandable, without changing their unique meaning. (6) Guided questions were constructed using a graduated prompt approach (Campione \& Brown, 1987). The questions were constructed with accordance to the extended conceptual base theory (Honeck, 1997), according to which a graduated cognitive effort is essential for proverbial understanding. This cognitive process involves creating assumptions, automatic processes, and solutions. In this process, five progressive stages are involved, each stage corresponds to a conceptual component of proverbial understanding administered in a graduated prompt approach: (a) Verbal understanding, (b) Analogical mapping, (c) Metaphorical mapping, (d) Understanding the proverb and its context, and (e) Application. These components are considered as progressive for understanding of the proverb. (7) A preliminary study on kindergarten $(n=40)$ and Grade 1 children $(n=111)$ conducted by Tzuriel and Valdman (2009) indicated satisfactory validity and reliability.

In this study, because of its relative length and to adjust for attention span, the test was shortened to seven proverbs, based on a pilot study on 20 children with HFA. These proverbs were chosen to adjust to the children's level of understanding of the themes. The four deleted items had relatively complex grammatical structure and include objects that are relatively remote from the child's experiential world. An example of the proverb "Honey is sweet, but the bee stings" and the accompanied questions according to the five components (steps) are presented in the following (Figure 1):

Dana's friend is Tami. Dana loves to play with her, but sometimes, Tami hits Dana. Dana's mother said, "I don't want you to be a friend with Tami." "But mammy, I love to play with Tami," answered Dana. "You have enough friends to play with them. You don't need her plays and not her hits ... Honey is sweet, but the bee stings." 


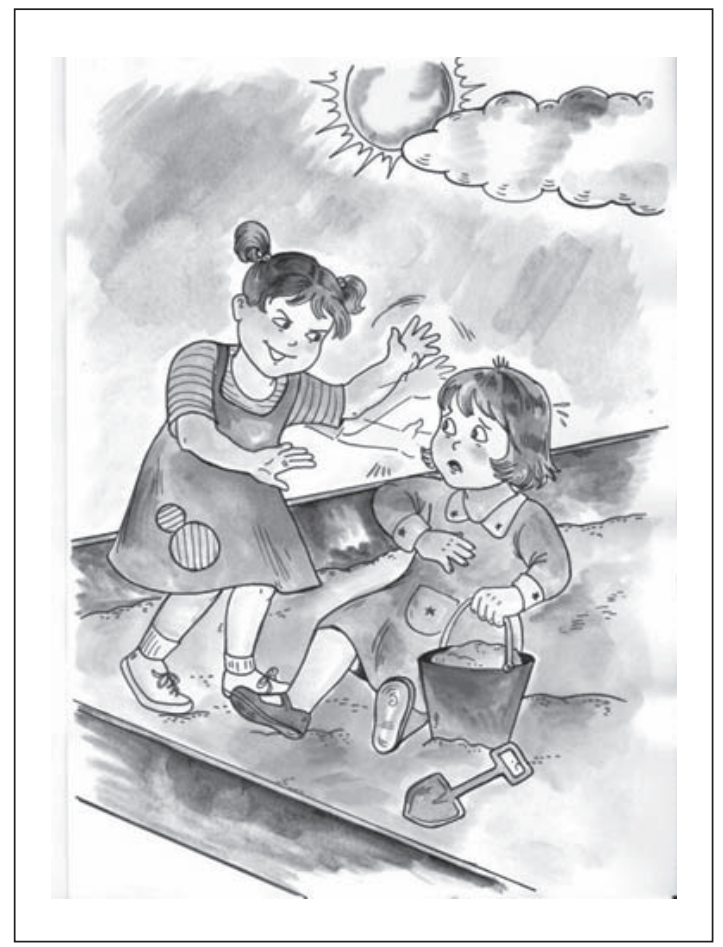

FIGURE 1. Example of Proverbial Understanding Test (Tzuriel \& Valdman, 2009).

After presenting this story, the examiner asks the following set of graduated prompts; each corresponds with the test's components. The child's response is recorded, and an answer was given by the examiner before moving to the next component:

a. Verbal understanding: What is honey? What is sting? Who has honey and stings?

b. Analogical mapping: Just as honey is sweet, but the bee stings, in the story that I told you, we don't want from the (plays) and not from the (hits of the child).

c. Metaphorical mapping: What is the honey in the story? What is sting in the story? Who behaves like a bee in the story?

d. Understanding the proverb and its context: What is the meaning of "Honey is sweet, but the bee stings"? Why Tami hits when plays are fun?

e. Application: Where else your mother, or father, or everyone else may tell you "Honey is sweet, but the bee stings"?

It should be emphasized that several researchers proclaim that proverbial understanding is ingrained in the culture and that transfer of a proverb across cultures might not reflect proverbial understanding capacity (e.g., Tavernier-Almada. 1999). However, many others argue that proverbial understanding is universal and that some proverbs are common across cultures (e.g., Mieder, 1993).

Scoring of the Children's Proverbial Understanding Test. In this study, the CPUT was scored by the graduated prompt approach. For each item, there are five progressive components; the child's 
answer for each component is given a score based on accuracy of the answer. For each component, a scale of five possible answers was constructed starting from full and accurate $($ score $=5)$ to lack of answer (score $=0$ ). After presenting the question for each component (stage), the child was given the answer, and the examiner moved to the next component until the last component of application representing full understanding of the proverb. This procedure was repeated in all items. Thus, for each item, there is a fixed protocol of prompts to reach the final stage of application. This novel procedure, which is a combination of graduated prompt and performance within each item, is different from the typical graduated prompt procedure where scoring is based on number of prompts. In this study, the number of prompts is fixed in each item and scoring is based on performance on each prompting stage. This procedure allowed us to adhere to the conceptual framework of attaining the proverb with an accurate scoring based on performance. Thus, the score for each component ranges between 0 and 5; for each item, between 0 and 25; and for the whole test, between 0 and 175 (7 items $\times 25)$. If the answer is not correct, the accurate answer is provided and the next question relating to the next component is presented. The same process continues to the last component of application.

Pearson correlations between the total score of each component (across all items) and the mean ratings based on three judges ranged between $.69(p<.01)$ and $.88(p<.01)$. Cronbach's alpha coefficient on the 11 original proverbs based on a sample of 111 TD children was .90 (Tzuriel \& Valdman, 2009). Principal components factor analysis with varimax rotation carried out on the 11 proverbs revealed only one factor (eigenvalue $=5.56$ ), which explained $51 \%$ of the variance. A second factor analysis with varimax rotation carried out on mean scores of the five test components (stages) revealed one factor (eigenvalue $=3.66$ ), which explains $73 \%$ of the variance. The CPUT was found to be correlated significantly (Tzuriel $\&$ Valdman, 2009) with age, .47 ( $p<.01)$; verbal ability, .77 $(p<.01)$; analogical reasoning, $.52(p<.01)$; and metaphorical construction, $.66(p<.01)$.

Children's Metaphorical Construction Test. The Children's Metaphorical Construction Test (CMCT; Tzuriel, Yosef, \& Valdman, 2008) is aimed at assessing metaphorical construction of elementary school children. Each metaphor is composed of three cards drawn with the metaphor's objects: origin object, target object, and a related-to-origin object (Figure 2). The test is composed of 2 example problems and 13 test problems. Children are asked to express

\section{The sun is like the lamp in the sky}
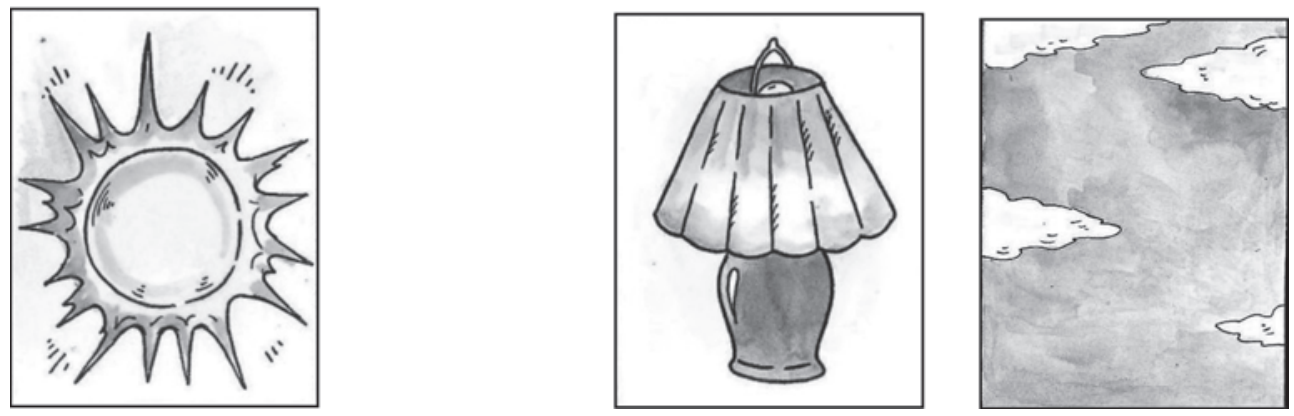

FIGURE 2. Example of an item from the Metaphorical Construction Test (Tzuriel et al., 2008). 
each metaphor while being introduced to the three cards of the item placed in a mixed order. In case the child could not express the metaphor, hints were provided based on the graduated prompt procedure. The principle behind the graduated prompt procedure is basically to help the child gradually until he or she constructs the metaphor. Mediation in this approach is delivered by a set of predetermined hints that range from general to specific. The examiner stops providing hints when the child reaches the level of independent task solution. The amount of aid needed to solve the problem is taken as an indication of the subject's zone of proximal development. The outcome measure in the graduated prompt approach is not the amount of improvement in the child's performance, but rather the amount of mediation needed to reach a specified criterion (Resing, 1997). The score for each metaphor ranges from 0 to 9 based on the amount of mediation required to provide the answer. For example, a maximal score of 9 points is given when the child expresses the metaphor independently with no mediation. If the child does not express the metaphor, a small hint is given, that is, an object's name. If the child expresses the metaphor after the first hint, a score of 8 points is given. The graduated prompt continues in a structured progressive procedure of nine steps; after each step of mediation, the score is reduced by 1 . Pearson's correlation between the CMCT and verbal ability test (MAASE) in the current sample was .62 $(p<.05)$. The CMCT reliability coefficient based on a sample of 53 children was .97. (Tzuriel \& Valdman, 2009).

Children's Conceptual and Perceptual Analogical Modifiability Test-Constructions Analogies Version. The Children's Conceptual and Perceptual Analogical Modifiability Test-Construction Analogies Version (Tzuriel, 2002) is composed of two sets of problems, conceptual and perceptual; each set contains nine problems: One example problem and eight test problems. In each problem, the child is presented with six cards, in a mixed order, and is asked to build an analogy with only four cards formatted in a $2 \times 2$ pattern, sorting out two distracting cards. The distracting cards contain stimuli that are related to the analogy but are not part of it. The distracting cards are based on categorical, part-whole, or associative relation. The child is presented first with an example problem in which the principles of analogy construction strategies are demonstrated. Examples of perceptual and conceptual construction analogies are presented in Figures 3 and 4, respectively. In each problem, the child has to choose four cards and construct them in the classical analogy format (i.e., A:B :: C:D). The construction analogies were administered by a clinical/educational version of administration (Tzuriel, 1997, 2001), using the graduated prompt procedure. The score for each problem ranges from 6 to 0 based on the amount of mediation required to provide the answer. A maximal score of 6 points is given when the child constructs the analogy independently with no mediation. If the child does not solve the problem, a hint is given (i.e., the A and B terms of the analogy are shown with no verbal explanation). If the child solves the problem after this hint, a score of 5 points is given. The graduated prompt continues in a structured progressive procedure of five steps; in each step of mediation, the score is reduced by 1 . If the child does not solve the problem after getting the mediation of the previous steps, a score of 0 is given.

The reliability of the construction analogies was studied previously on four samples of kindergarten children (Tzuriel, 2002, 2007; Tzuriel \& Flor-Maduel, 2010; Tzuriel \& George, 2009). In all samples, children were administered the closed analogies prior to the construction analogies. Cronbach's alpha reliability coefficients for the conceptual analogies ranged between .83 and .87 and for the perceptual analogies, between .84 and .87 .

Spoken Language Test. The verbal ability of children was examined by the Spoken Language Test developed (in Hebrew) to assess semantic language abilities of children aged 5-11 years. 

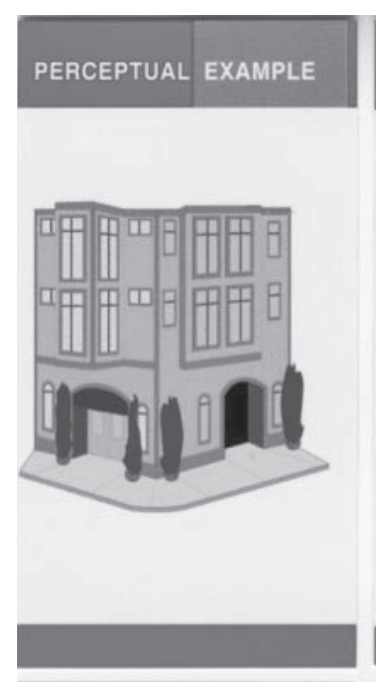

PERCEPTUAL EXAMPLE
PERCEPTUAL EXAMPLE
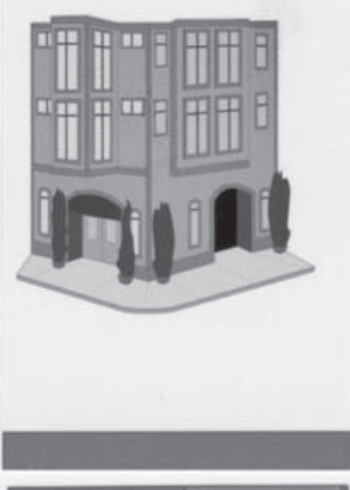

PERCEPTUAL EXAMPLE
PERCEPTUAL EXAMPLE
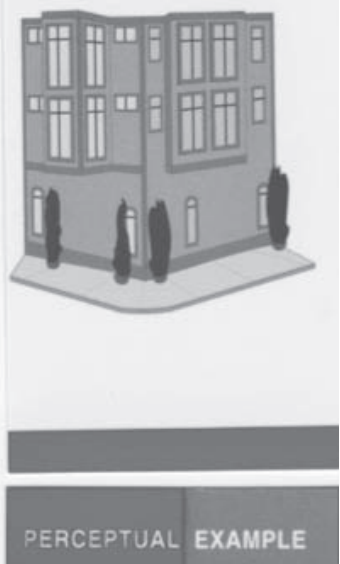
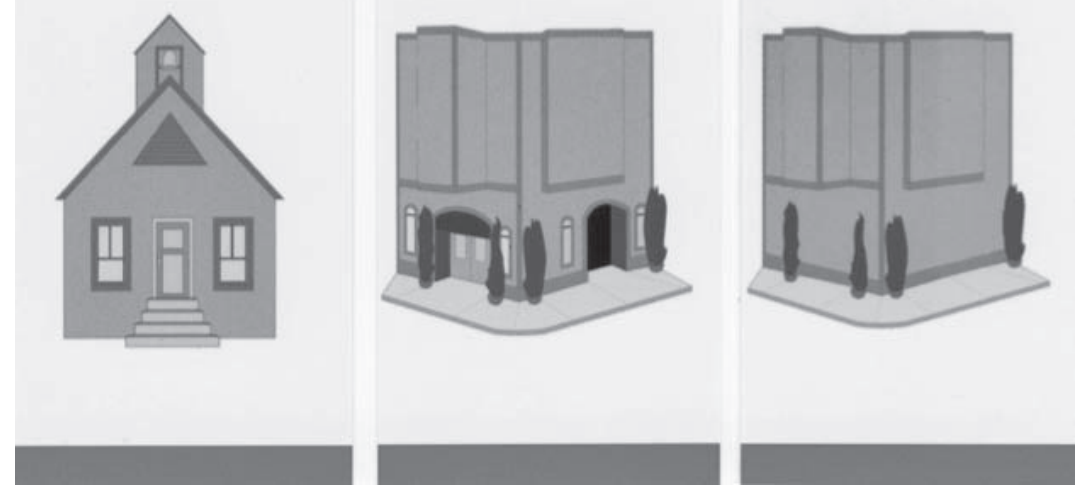

FIGURE 3. Example item of construction perceptual analogy from the Children's Conceptual and Perceptual Analogies Modifiability (Tzuriel, 2002, 2007).

In this study, the children were administered the new version of the test (Rom, Morag, \& Peleg, 2007), which is suited to the children's gradual increase of semantic abilities in those ages. The test includes two pretests: (a) naming objects and (b) verbs (e.g., provide the verbal noun related to the objects). Additional five subtests are Categories, Resemblance, Difference, Ambiguity, and Definitions. In Categories children are presented verbally with 10 categories (e.g., holidays, jewelry) of which the children are asked to name three different objects belonging to each. In Resemblance, children are verbally presented with 10 object pairs (e.g., car and bus) and are asked to name the similarity between the objects. In Difference, children are introduced to the same 10 object pairs and are asked to name a difference between the objects. In Ambiguity, children are introduced to 10 homonyms (ambiguous words that are written and pronounced in the same way) and are asked to name the meaning of each word 


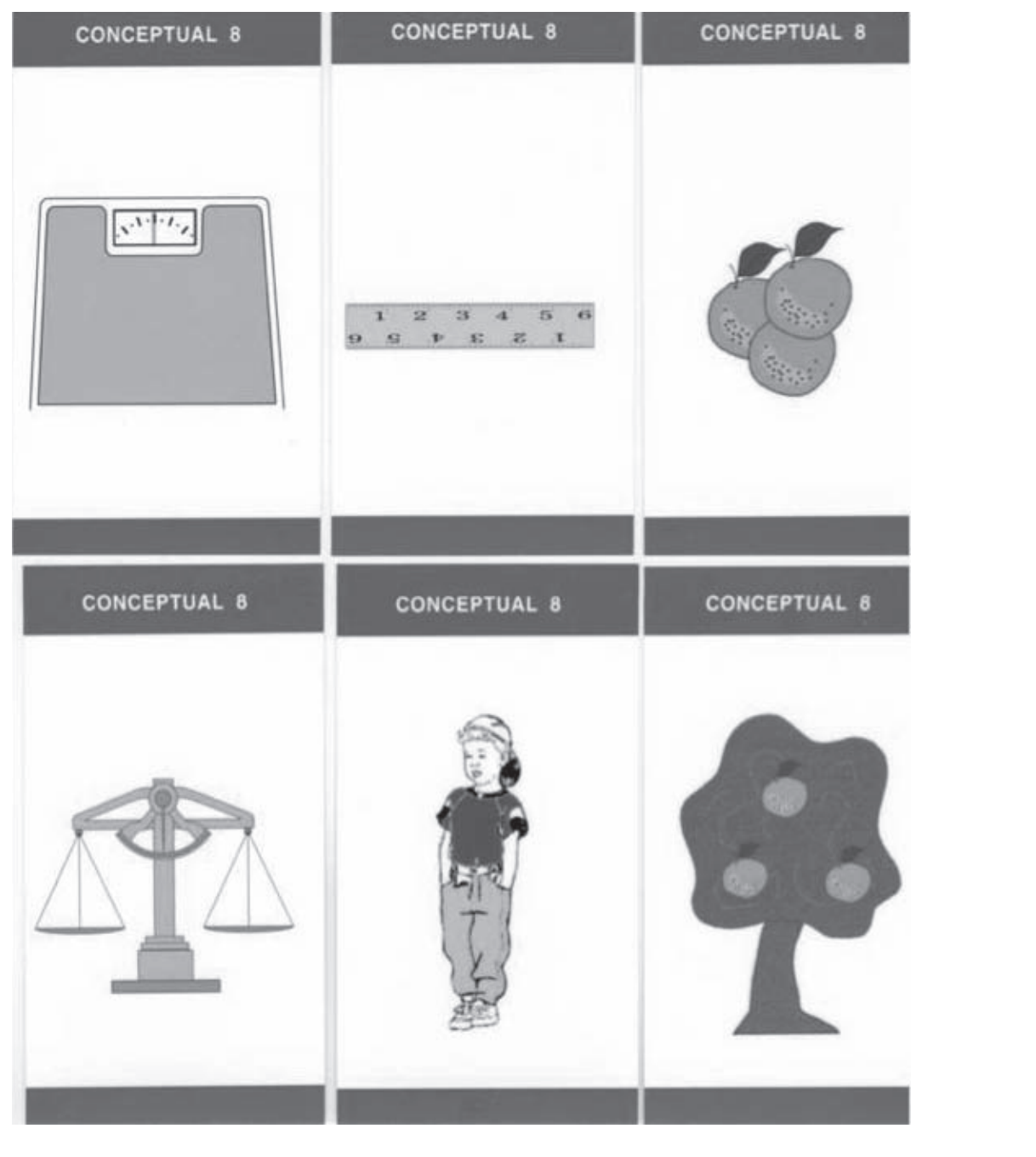

FIGURE 4. Example item of construction conceptual analogy from the Children's Conceptual and Perceptual Analogies Modifiability (Tzuriel, 2002, 2007).

in a specific context. In Definitions, children are verbally presented with five objects and are asked to provide specific descriptions related to different features (e.g., size, shape, or color) for each object. In each subtest, a correct full answer is given a score of 2, a partial answer a score of 1 , and an incorrect answer or no answer a score of 0 . Scores for each of the subtests range from 0 to 20, with higher scores reflecting a higher linguistic level. Pearson's correlation between the MAASE subtests and children's Auditory Association (ITPA) subtest were .65 and above (Fisher, 1975). The reliability coefficients based on previous sample were above .60 (Tzuriel \& Valdman, 2009).

The Rule Shift Card Test. The Rule Shift Card (RSC) test (Wilson, Alderman, Burgess, Emslie, \& Evans, 1996) is a subtest of Wisconsin Card Sorting Test (WCST; Heaton, Chelune, Talley, Kay, \& Curtiss, 1993). The WCST is a measure of abstract reasoning that requires the ability to shift strategies. The WCST has been validated in individuals with autism (Ozonoff, 
1995) and has previously been used to measure shifting in children with autism, with and without mild intellectual disability (ID; e.g., Robinson, Goddard, Dritschel, Wisley, \& Howlin, 2009; Visser, Berger, Van Schrojenstein Lantman-De Valk, Prins, \& Teunisse, 2015). Participants are presented with a series of 20 red and black cards turned over one at a time. In the first stage of the task, participants have to act according to the rule, "Say yes to red and no to black." In the second stage, the rule changes- "Say yes if the card is in the same color as the last one, otherwise, say no." The second stage taps the participants' cognitive flexibility because they have to delay the familiar reaction and create a different reaction. Kendall's tau correlations of WCSTs were .65-.75 ( $p<.001$; Smith-Seemiller, Arffa, \& Franzen, 2001). Reliability coefficients based on previous samples were above .60 (Heaton et al., 1993; Rajendran \& Mitchell, 2007).

Children's Local/Central Coherence Test. The Children's Local/Central Coherence Test (CLCCT), which is based on the WCCT (Frith, 1989; Frith \& Happé, 1994), is aimed at measuring LCC processing (Jolliffe \& Baron-Cohen, 1999). The test developed for this study by Tzuriel and Groman (2013) was adapted for children aged 5-11 years by simplifying the level of vocabulary and using age-appropriate context. The test is composed of 1 example item and 10 test items. The tasks require preliminary basic reading comprehension and memorizing specific details incorporated in a short story. In each item, children should read a description of a situation followed by a question and three possible options for an answer. Following introduction of each situation and the options for an answer, children are asked to think about a statement that bridges the sentences in the most accurate way. For example, children were asked the following: "Dan was in a hurry to the candy shop. A few minutes afterwards, he was smiling to himself. Dan was smiling because: 1 . He met friends on his way to the shop. 2. He bought a candy. 3. He met a friend who gave him a candy." Each correct answer was given a score of 1 , and the total possible score is 10 . The test's validity was examined in several steps. In the first step, a pilot study was carried out on a sample of TD children $(n=10)$ and children with HFA $(n=10)$, aged $5-7$ years. The purpose was to examine the fitness of items to young children and especially to children with HFA. The findings revealed as expected that TD children scored higher than children with HFA; the range of scores for the TD group was 6-9 $(M=7.90, S D=2.07)$ as compared with $4-8(M=5.90, S D=1.19)$ in the HFA group. In the second step, a group of five experienced clinicians examined the items for fitness to children with HFA. Based on their recommendations, it was decided to change the test procedure and give children first the opportunity to provide an answer, and only when no answer is given, the three answer options are provided for choice. An initiated answer gets a score of 2 and choice of a correct option a score of 1 ; the maximal score is 20 . In the third step, we applied a second pilot study using the modified procedure on a sample of TD children $(n=15)$ and children with HFA $(n=15)$ aged $6-11$ years old. Cronbach's alpha reliability coefficient based on this sample was .85. Pearson's correlation between the CLCCT scores and items from the Global Local Processing Strategies (GLPS, Kimchi \& Palmer, 1982) based on a sample of 88 children was $.24(p<.05)$. In the current sample, principal factor analysis with varimax rotation showed that all test items are loaded on one factor (eigenvalue $=4.40$ ) explaining $44 \%$ of the variance. Cronbach's alpha coefficient based on the current sample was .88.

Theory of Mind Tests. In this study, we used two tests. The first test, Sally and Anne Test (Wimmer \& Perner, 1983) assesses children's understanding of first-order belief. The test consists of a comic-strip story in which Sally and Ann are first introduced as friends. Sally with a basket in front of her and Anne with a box. Then, Sally is shown placing a ball in the 
basket and leaving the room. Anne is then shown taking the ball from the basket and placing it in the box. Next, Sally enters the room and children are asked, "Where will Sally look for her ball?" Children who point to the previous location of the ball (basket) pass the task because they acknowledge Sally's false belief (score $=1$ ). Children who point the current location of the ball (box) fail the task by not considering Sally's false belief (score $=0$ ).

The John and Mary Test. This test assesses children's understanding of second-order belief. The test is an acted story in which two school friends (John and Mary) are independently informed about an object's transfer to a new location (an ice cream van moves from the park to the church). The van's driver then tells John that he travels to the school after Mary left from her home. On his way to school, the driver meets Mary and tells her about the change of location, but John is not aware of it. When John comes to Mary's home to tell her about the change of location, he is told that Mary already went to buy an ice cream. Children are then asked three questions: "Where will John look for Marry?" "Where is Mary?" and "Where is the ice cream van?" Scores on this test are only for the first question, which examines understanding of second-order belief (passed $=1$ ). Pearson's correlations between the two ToM tests range between $.45(p<.05)$ and $.55(p<.05)$. Cronbach's alpha coefficient based on a sample of 70 participants was .85 (Baron-Cohen, 1989; Perner \& Wimmer, 1985).

\section{Procedure}

All children were administered the tests in a quiet, isolated room at school or at home. The examiners reported no difference in children's motivation or in testing procedure at home or in school. Tests were administered individually in seven meetings, with respect to the child's comfort and ability. The tests of MAASE, construction analogies, and the CMCT were administered in the first three sessions, respectively. The CPUT was administered in the fourth session, and the EF, CLCCT, and ToM tests were administered in the fifth to seventh sessions, respectively. The examiners were graduate students in a special education program who had few years of teaching experience with children with HFA. All examiners were trained to administer the tests for $8 \mathrm{hr}$.

\section{RESULTS}

\section{Differences Between Children With High-Functioning Autism and Typically Developed Children in Proverbial Understanding}

The means and standard deviations of proverbial understanding scores in the HFA and TD groups and $F$ statistics are presented in Table 1. The HFA group scored significantly lower than the TD group on each stage of the Proverbial Understanding Test as well as on the total score, thus confirming our expectation. It is interesting to note that the highest group difference was found on the Application component $\left(\eta^{2}=.45\right)$ of the Proverbial Understanding Test. The ratio between Verbal understanding (first step of the test and mostly the easiest) and Application (last step of the test and the most difficult) is much lower in the HFA group (.32) than in the TD group (.76; a difference which will be discussed later).

To compare children's improvement from the start to the end of the DA procedure, we created compiled subscores of the first three items (pre, Time 1) and the last four items (post, Time 2). The first three items were similar to the last four items in terms of grammatical 
TABLE 1. Proverbial Understanding Scores in the High-Functioning Autism and Typically Developing Groups

\begin{tabular}{|c|c|c|c|c|c|c|}
\hline \multirow[b]{2}{*}{ Proverbial Understanding } & \multicolumn{2}{|c|}{ HFA } & \multicolumn{2}{|c|}{ TD } & \multirow[b]{2}{*}{$F(56)$} & \multirow[b]{2}{*}{$\eta^{2}$} \\
\hline & $M$ & $S D$ & $M$ & $S D$ & & \\
\hline Total & 3.57 & 0.64 & 4.61 & 0.41 & 59.17 *冰火 & .49 \\
\hline Verbal understanding & 4.48 & 0.66 & 4.92 & 0.18 & $12.99 * * *$ & .17 \\
\hline Analogical mapping & 0.28 & 0.67 & 4.94 & 0.11 & $29.84 * * * *$ & .32 \\
\hline Metaphorical mapping & 4.18 & 0.94 & 4.89 & 0.18 & $17.60 * * * *$ & .22 \\
\hline Understanding the proverb & 3.46 & 1.06 & 4.56 & 0.60 & $25.61 * * * *$ & .29 \\
\hline Application & 1.23 & 1.44 & 3.73 & 1.31 & $51.05 * * * *$ & .45 \\
\hline
\end{tabular}

$\mathrm{HFA}=$ high-functioning autism; TD $=$ typically developing.

$* * p<.01 . * * * p<.001$.

syntax (i.e., time components, quantitative comparison, and cause-effect relationships) and degree of remoteness of objects from the child's experiential world. It should be emphasized that our data are based on the graduated prompt approach in which for each item, there is a fixed protocol of prompts to reach the final stage of application. This technique is different from both the typical pre- to postteaching model of DA where the criterion variable is only performance score and from the conventional graduated prompt model where the number of prompts is considered. We used the graduated prompt when we applied the five conceptual stages (components) for each item, but we scored the performance on each component. We expected therefore that the number of prompts will decrease significantly from the first to last items of the test as the child learns progressively the essence of proverbs and metaphors.

Because we were interested in examining interactions of Group with Time and Component variables, a repeated measures multivariate analysis of variance (MANOVA) of Group $\times$ Time $\times$ Component $(2 \times 2 \times 5)$ was carried out with Group as between factor, Time and Component as within factors, and proverbial understanding scores as dependent variables. The analysis yielded significant main effects of Group, $F(1,61)=58.93, p<.001, \eta^{2}=.49$; Component, $F(4,61)=108.76, p<.001, \eta^{2}=.64$; and Time, $F(1,61)=16.25, p<.001$, $\eta^{2}=.21$. These findings indicate, as expected, that children with HFA received lower scores than TD children, that the scores decreased with increase of the component's difficulty level, and that there was an improvement from the pre to the post items.

The main effects however were modified by two significant interactions of Group $\times$ Component, $F(4,61)=17.98, p<.001, \eta^{2}=.23$, and Time $\times$ Component, $F(4,61)=4.54, p<.001$, $\eta^{2}=.07$. Theseinteractions are depicted in Figures 5 and 6. Posthoc analyses show that the Group $\times$ Component interaction (Figure 5) derives mainly from the Application component. Group differences on each components were as follows: (a) Verbal understanding, $F(1,61)=13.44$, $p<.001, \eta^{2}=.18$; (b) Analogical mapping, $F(1,61)=29.78, p<.001, \eta^{2}=.33$; (c) Metaphorical mapping, $F(1,61)=17.85, p<.001, \eta^{2}=.23$; (d) Understanding the proverb and its context, $F(1,61)=25.43, p<.001, \eta^{2}=.29$; and (e) Application, $F(1,61)=49.81, p<.001$, $\eta^{2}=.45$. The findings show, in general, that group differences increase with increase of the component's difficulty level and that group difference in Application component $\left(\eta^{2}=.45\right)$ is much higher than in the other components $\left(\eta^{2}=.18\right.$ to .29). 


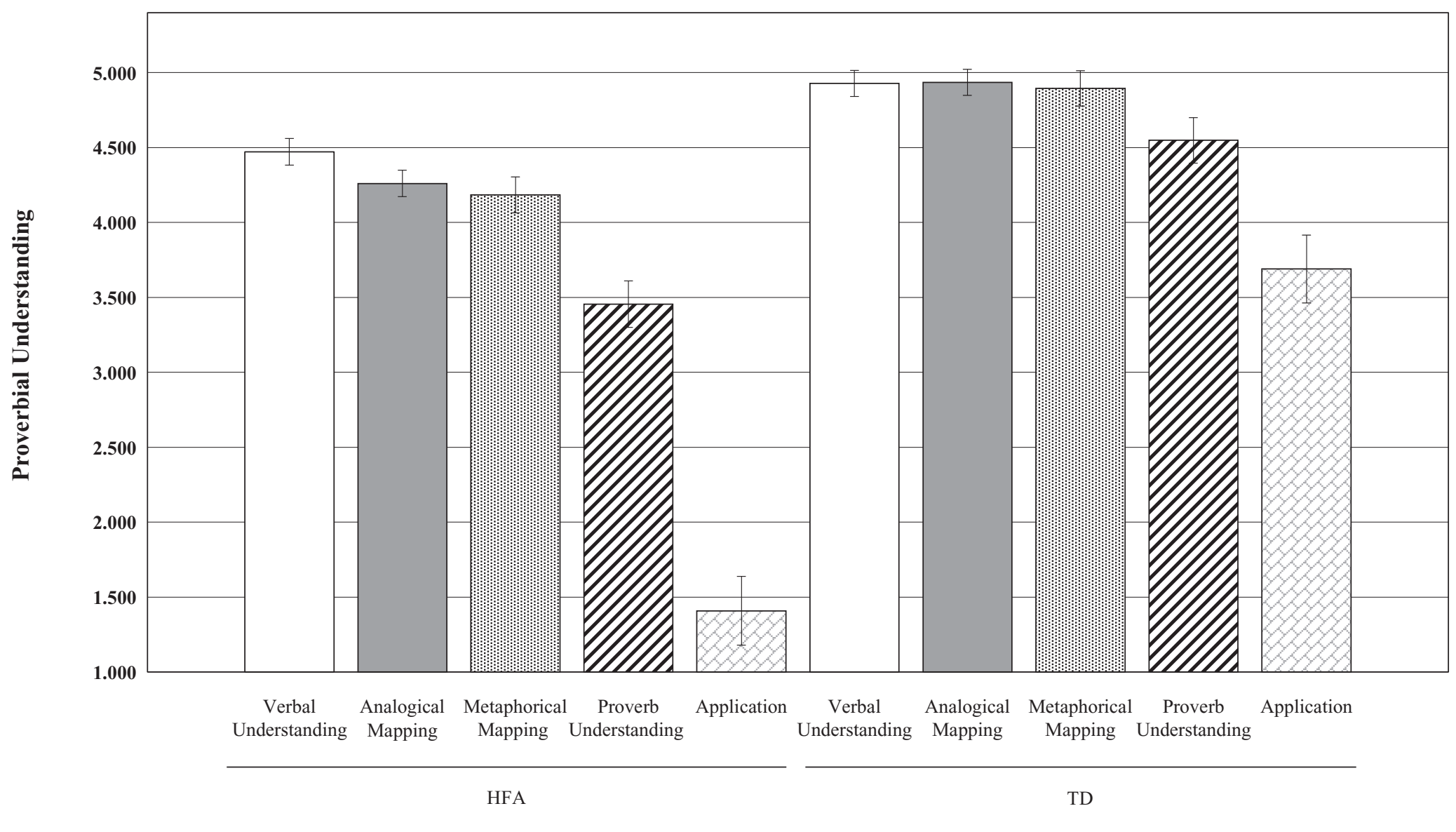

FIGURE 5. Proverbial understanding components in the high-functioning autism and typically developing groups. 


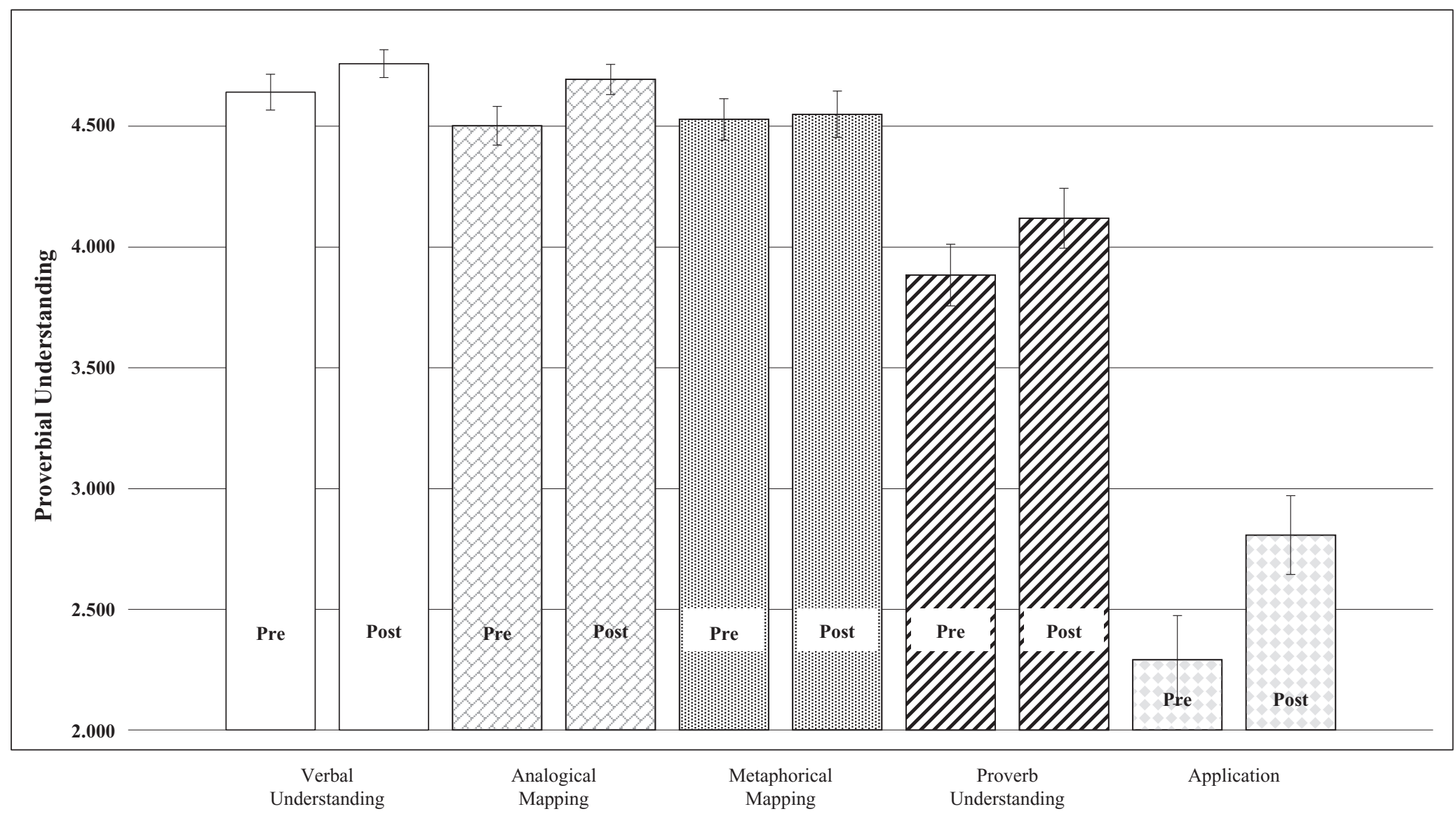

FIGURE 6. Proverbial understanding components in pre and post stages. 
Post hoc analysis of the Time $\times$ Component interaction (Figure 6 ) indicates that it derives mainly from three components: (a) Verbal understanding, $F(1,61)=6.60, p<.05, \eta^{2}=.10$; (b) Analogical mapping, $F(1,61)=6.70, p<.001, \eta^{2}=.10$; and (c) Application, $F(1,61)=16.33$, $p<.001, \eta^{2}=.21$. The highest pre to post improvement was found on Application.

\section{Differences Between Children With High-Functioning Autism and Typically Developed Children in Metaphorical Construction}

To compare group differences as well as children's improvement from the start to the end of the DA process in both groups, we created compiled scores of the first five items (pre, Time 1) and the last six items (post, Time 2). As in the Proverbial Understanding Test, the data are based on the graduated prompt approach in which for each item, there is a fixed protocol of nine prompts to reach the answer. If the child solves the problem with no prompts, a maximal score of 9 is given; the more prompts are given, the lower is the child's score. A repeated measures analysis of variance (ANOVA) of Group $\times$ Time $(2 \times 2)$ yielded significant main effects of Group, $F(1,60)=31.35, p<.001, \eta^{2}=.34$, and Time, $F(1,60)=12.47, p<.001, \eta^{2}=.17$. These findings indicate that the TD group scored higher $(M=10.01, S D=1.56)$ than the HFA group $(M=7.16, S D=2.76)$ and that performance in the last six items was higher $(M=9.08, S D=2.92)$ than in the first five items $(M=8.18, S D=2.92)$.

\section{Analogical Reasoning, Executive Function, Local/Central Coherence, Verbal Ability, and Theory of Mind in the High-Functioning Autism and Typically Developing Groups}

The means and standard deviations on analogical reasoning, subtest-I of EF, LCC, verbal ability, and ToM in the HFA and TD groups are presented in Table 2. The findings show that, as expected, TD children scored significantly higher on analogical reasoning and LCC than children with HFA. No significant group differences however were found on verbal ability, except for the Naming subscale (from MAASE test) where children with HFA scored higher than TD children. The findings also reveal that TD children scored significantly higher than children with HFA in the RSC test, ToM general score, and John and Mary subtest.

\section{Correlations Between Proverbial Understanding and Metaphorical Construction, Verbal Ability, Analogical Reasoning, Executive Function, Theory of Mind, and Local/Central Coherence}

Pearson correlations between proverbial understanding and metaphorical construction, verbal ability, analogical reasoning, EF, ToM, and LCC was carried out separately in the HFA and TD groups. The analysis revealed a different pattern of correlations in the two groups (Table 3). In the HFA group, positive significant correlations were found between proverbial understanding and analogical reasoning (perceptual), EF, ToM, and LCC. Verbal ability has emerged with the strongest correlation in the HFA group $(r=.51)$ as compared with no correlation in the TD group $(r=.00)$. In the TD group, proverbial understanding was highly correlated with metaphorical construction $(r=.79)$ and LCC $(r=.70)$ as compared with low $(r=.06)$ and medium correlation $(r=.39)$ in the HFA group. Fisher $Z$ analyses showed that the difference in correlation pattern appears only for verbal ability, metaphorical construction, and EF. 
TABLE 2. Verbal Ability, Analogical Reasoning, Executive Memory, Theory of Mind, and Local/Central Coherence in the High-Functioning Autism and Typically Developing Group

\begin{tabular}{|c|c|c|c|c|c|}
\hline \multirow[b]{2}{*}{ Test } & \multicolumn{2}{|c|}{ HFA } & \multicolumn{2}{|c|}{ TD } & \\
\hline & $M$ & $S D$ & $M$ & $S D$ & \\
\hline \multicolumn{6}{|l|}{ Verbal ability } \\
\hline Total & 0.69 & .07 & .71 & .05 & $F_{(1,50)}=1.03$ \\
\hline Naming & 0.99 & .02 & 0.96 & .06 & $F_{(1,50)}=5.56^{*}$ \\
\hline Verbs & 0.98 & .04 & 0.99 & .01 & $F_{(1,50)}=1.95$ \\
\hline Categories & 1.81 & .15 & 1.87 & .17 & $F_{(1,50)}=2.19$ \\
\hline Resemblance & 0.88 & .20 & 0.95 & .09 & $F_{(1,50)}=2.87$ \\
\hline Difference & 0.93 & .10 & 0.92 & .18 & $F_{(1,50)}=0.48$ \\
\hline Ambiguity & 1.82 & .20 & 1.91 & .16 & $F_{(1,50)}=2.91$ \\
\hline Definitions & 1.10 & .49 & 1.13 & .36 & $F_{(1,50)}=0.03$ \\
\hline \multicolumn{6}{|l|}{ Analogical reasoning } \\
\hline Total & 5.10 & .70 & 5.59 & .30 & $F_{(1,60)}=12.57 * *$ \\
\hline Perceptual & 5.21 & 0.91 & 5.85 & 0.35 & $F_{(1,60)}=13.51 * *$ \\
\hline Conceptual & 5.00 & 0.81 & 5.33 & 0.41 & $F_{(1,60)}=4.05^{*}$ \\
\hline \multicolumn{6}{|l|}{ EF } \\
\hline Total & 17.84 & 1.73 & 18.43 & 1.73 & $F_{(1,61)}=1.87$ \\
\hline Subtest 1 & 18.94 & 1.58 & 19.81 & 0.53 & $F_{(1,61)}=8.75 * *$ \\
\hline Subtest 2 & 16.75 & 2.54 & 17.06 & 0.35 & $F_{(1,61)}=0.17$ \\
\hline \multicolumn{6}{|l|}{ ToM } \\
\hline Total & 0.46 & 0.38 & 0.75 & 0.35 & $F_{(1,60)}=9.03 * *$ \\
\hline Sally and Anne & 0.55 & 0.50 & 0.78 & 0.42 & $F_{(1,60)}=3.96$ \\
\hline Local/Central Coherence & 9.38 & 3.69 & 16.03 & 3.65 & $F_{(1,61)}=7.17 * * *$ \\
\hline
\end{tabular}

$\mathrm{HFA}=$ high-functioning autism; $\mathrm{TD}=$ typically developing; $\mathrm{EF}=$ executive function; $\mathrm{ToM}=$ theory of mind.

$* p<.05 . * * p<.01 . * * * p<.001$.

TABLE 3. Correlations Between Proverbial Understanding and Verbal Ability, Analogical Thinking, Metaphorical Construction, Executive Memory, Theory of Mind, and Local Coherence in the High-Functioning Autism and Typically Developing Groups

\begin{tabular}{lccc}
\hline & \multicolumn{2}{c}{ Proverbial Understanding } & \\
\cline { 2 - 3 } Test & HFA & TD & Fisher Z \\
\hline Verbal ability & $.51 * *$ & -.00 & $2.03 *$ \\
Perceptual analogies & $.34 *$ & .06 & $n s$ \\
Conceptual analogies & -.07 & -.05 & $n s$ \\
Metaphorical construction & .06 & $.79 * * *$ & $-3.64 * * *$ \\
EF & $.35 *$ & $-.44 * *$ & $3.02 * *$ \\
ToM & $.31 *$ & -.04 & $n s$ \\
Local/central coherence & $.39 *$ & $.70 * * * *$ & $n s$ \\
\hline
\end{tabular}

$\mathrm{HFA}=$ high-functioning autism; $\mathrm{TD}=$ typically developing; $\mathrm{EF}=$ executive function; $\mathrm{ToM}=$ theory of mind.

$* p<.05 . * * p<.01 . * * * p<.001$. 
TABLE 4. Prediction of Proverbial Understanding by Cognitive Abilities, Executive Memory, Local Coherence, and the Ability to Understand False Belief in Others in Each Group

\begin{tabular}{lcccc}
\hline Test & HFA $(\beta)$ & TD $(\beta)$ & $R$ & $R^{2}$ \\
\hline Verbal ability & .57 & - & .69 & .48 \\
Local/global coherence & .46 & - & & \\
Metaphorical construction & - & .73 & .85 & .72 \\
Executive memory & - & -.30 & & \\
\hline
\end{tabular}

HFA $=$ high-functioning autism; TD $=$ typically developing.

$* p<.05 . * * p<.01 . * * * p<.001$.

\section{Regression Analysis of Proverbial Understanding by Verbal Ability,} Analogical Reasoning, Metaphorical Construction, Executive Function, Theory of Mind, and Local/Central Coherence

To examine the relative strength of verbal ability as compared with other cognitive factors in predicting proverbial understanding in the HFA and TD groups, we conducted a separate stepwise regression analyses in each group (Table 4). The findings show that in the HFA group, verbal ability (.54) and local/global coherence (.46) have emerged as significantly predicting proverbial understanding, whereas in the TD group, metaphorical construction (.73) and EF (-.30) significantly predict proverbial understanding.

\section{DISCUSSION}

The findings have shown, as expected that TD children perform better in proverbial understanding and metaphorical construction than children with HFA. These findings are supported by previous findings showing that children in the autistic spectrum have significantly lower figurative language ability than TD children (e.g., Dennis et al., 2001; MacKay \& Shaw, 2004; Rundblad \& Annaz, 2010). A possible explanation for the group differences in proverbial understanding might be related to the role of metaphorical construction for enhancement of proverbial understanding. Lakoff and Turner (1989) have suggested that proverbial understanding is carried out by a metaphorical mapping principle based on an automated activation of a metaphor existing in the proverb's basis. The ability to interpret proverbs in different ways derives from the ability to map the metaphorical knowledge existing in proverbs to diverse target fields (Gibbs 2008; Gibbs \& Beitel, 1995). Support for this explanation may be found in the findings of this study showing significantly lower scores of the HFA group, as compared with the TD group, on the Metaphorical Construction Test and the metaphorical mapping component of the Proverbial Understanding Test (see Table 1). Another factor that might contribute to the lower proverbial understanding of children with HFA might be attributed to the relatively central coherence processing found to be diminished in individuals with autism (Frith, 1989). Support for this relation might be found in the findings of this study showing significant correlations between global/local coherence and proverbial understanding in both TD $(r=.70)$ and HFA $(r=.39)$ groups (see Table 3). Proverbial understanding requires construction of new meanings and expanding them to new varied events 
(Honeck, 1997; Sperber \& Wilson, 1986). This ability requires a central coherence type of processing so that the child can capture the essence of the proverb.

The role of global/local coherence and metaphorical construction for proverbial understanding contradicts the conclusions of Gernsbacher and Pripas-Kapit (2012) who have stated that individuals with autism who have difficulty in language comprehension, might have as well difficulty in comprehending metaphoric, idiomatic, inferential, potentially ambiguous, or otherwise complex language. They emphasize that the same goes for nonautistic persons and that children who have a language disability, regardless of whether they have autism or not, have difficulty with metaphoric language. This argument is supported by Norbury's (2005a, 2005b) conclusion, based on several studies, that comprehension ability per se is "the most important determinant of success" in comprehending idioms (Norbury, 2004, p. 1190). The findings of our study do not rule out the importance of verbal ability in determining figurative language. The correlation (see Table 3) and the regression analyses (see Table 4) point clearly to its strong relation with proverbial understanding, especially in the HFA group. However, our findings clearly indicate that figurative language may be attributed as well to unique difficulties in metaphoric construction and the tendency for local coherence.

Of most interest is the higher performance level of the TD group over the HFA group across all of the proverbial understanding components but especially on Application, considered to be the most difficult component. As can be seen in Table 1, the ratio between Verbal understanding (first step of the test and mostly the easiest) and Application (last step of the test and the most difficult) is much lower in the HFA group (.32) than in the TD group (.76). The meaning of this finding is that, from all components of the test, children in the HFA group confront difficulties especially in the Application component. Application requires a transfer of the concrete meaning of the proverb to a different context, moving flexibly from the contextual understanding of the specific situation to varied situations where the same meaning can be applied as well. Similarly, the significant Group $\times$ Component interaction shows (see Figure 5) that it derives mainly from the Application component found to be significantly lower in the HFA than in the TD group. This difference is congruent with clinical and research findings indicating unique difficulties of applying subtle meanings of figurative language beyond the specific and concrete situations among children in the autistic spectrum (Joliffe \& Baron-Cohen, 1999; Kerbel \& Grunwell, 1998; Melogno et al., 2012; Persicke et al., 2012; Wang, Lee, Sigman, \& Dapretto, 2006; Worth \& Reynolds, 2008). It should be emphasized that the graduated prompt approach used in this study is characterized by "light" mediation. Nevertheless, it was found to improve the performance of initial components in children with HFA. It is plausible to assume that with a stronger "dose" of mediation, performance on Application component would be enhanced and the ratio with easier components of the proverbial understanding will increase.

The use of DA procedure in this study revealed that teaching within testing facilitated the children's proverbial understanding and that the improvement from the first to last items was similar for both groups and even slightly better in the HFA group than in the TD group. Previous studies showed that children with learning difficulties benefit significantly more from teaching within the test procedure than children with no learning difficulties (e.g., Haywood \& Lidz, 2007; Haywood \& Tzuriel, 2002; Sternberg \& Grigorenko, 2002; Tzuriel, 2001). Teaching in these studies was much heavier than the graduated prompt approach used in this study. In further research, we propose to use comparatively different teaching strategies (e.g., mediated learning experience vs. graduated prompt) and examine their effect on modifiability of figurative language and specifically modifiability of proverbial understanding. 
The interaction of Component $\times$ Time (see Figure 6) reveals that the differential preto postteaching changes derive mainly from three components, each reflecting a different phase of the mental act (Feuerstein et al., 1979): the input phase (i.e., Verbal understanding), the elaboration phase (i.e., Analogical mapping), and the output phase (i.e., Application). It should be noted that the highest improvements appear in output aspect of Application. Application of a proverb requires transferring the meaning of a proverb to context that represents the proverb in a real-life situation. The difficulty revealed by the children with HFA was in making this transition from cognitive understanding of the proverb to actual social context. Support for our findings may be found in studies showing that children with HFA demonstrate difficulties in flexibly applying principles and rules to social situations (Frith \& Happé, 1994; Peterson \& Bowler, 2000; Plaisted, 2001). The within-test mediation process thus was more effective in the Application component than in the others.

The findings on metaphorical construction showed, as expected, that the HFA group received significantly lower scores than the TD group. These findings are congruent with earlier findings showing higher metaphorical abilities among TD children than among children in the autistic spectrum (Dennis et al., 2001; MacKay \& Shaw, 2004; Rundblad \& Annaz, 2010). The difficulty of children in the autistic spectrum to grasp metaphors was attributed to a tendency to local processing (e.g., Frith, 1989) and to wrong interpretation in decoding a presented object (visual and auditory), as evidenced in the relatively low score of the HFA group on Verbal Ability component in the Proverbial Understanding Test. An incorrect decoding of an object presented in the metaphor might affect the whole perception of the metaphor. For example, in this study, some children with HFA interpreted a picture frame as a geometric figure (rectangle), thus focusing on geometric aspects of the picture rather than on its social contextual meaning.

Another explanation for differences in metaphorical understanding might be based on previous exposure to the objects presented in the metaphors. Processing of meaning of figurative language is explained in the literature by the graded salience hypothesis. In accordance with the graded salience hypothesis, linguistic processing is based on the most conventional, popular, predictable, or familiar meaning (e.g., Giora, 2003). Making mental relationships between components of a metaphor is a unique individual mode based on previous exposure to the components as well as on the analogical transformation required in the metaphor. The unique way of connecting between the metaphor's components is based also on chronological and mental age, on accumulated knowledge acquired with age, and on development of abstract skills (Nippold \& Sullivan, 1987). Thus, an early rich exposure to objects and their verbalization might affect the ability to process later metaphors based on these objects.

The correlations of proverbial understanding with the cognitive abilities showed a differential pattern in the HFA and TD groups (see Table 3). In the HFA group, verbal ability and EF were found significantly correlated (.51 and .35, respectively) with proverbial understanding as compared with the TD group (.00 and -.44 , respectively). The highest correlations of proverbial understanding in the TD group were with metaphorical construction (.79) and LCC (.70). It is reasonable to assume that children with HFA rely on verbal repertoire and EF, as represented by the RSC test, when processing figurative forms of language. These skills require a relatively lower level of abstract processing than metaphorical and central coherence skills found to be significantly correlated in the TD group.

The findings of the regression analysis portrayed a different picture of the relationship between proverbial understanding and the cognitive tests in the HFA and TD groups. In the 
HFA, group proverbial understanding was predicted positively by a combination of verbal ability $(\beta=.57)$ and LCC ( $\beta=.46)$, whereas in the TD group, it was predicted positively by metaphorical construction $(\beta=.73)$ and negatively by EF $(\beta=-.30)$. The significant prediction of proverbial understanding by metaphorical construction found in the TD children in the current research is supported by similar previous findings on a sample $(n=58)$ of Grade 1 children (Tzuriel \& Valdman, 2009). Understanding figurative forms such as proverbs and metaphors requires the ability to construct novel preferred meanings by pragmatic reasoning processes. In contrast, the negative prediction of proverbial understanding by EF in the TD group might be explained by the preference of using elastic abstract thinking strategies in interpreting figurative language forms. EF in RSC test reflects both cognitive flexibility (while shifting from one rule to another) and memory (while remembering the new rule). We might assume that children in the HFA group rely on memory ability while processing abstract forms of language, whereas in the TD group, this dimension restricts their abstract processing. EF are related in the literature to the ability to maintain a suitable pattern of problem solving to gain a future goal which includes behavior components such as planning, inhibition, stable management, organized exploring, and flexible thinking (Bauminger-Zviely, 2013; Martin \& McDonald, 2003; Ozonoff et al., 1991). Observing other EF such as inhibition and flexible thinking might provide an additional knowledge about the prediction of proverbial understanding by executive functions.

In this study, a primary condition for participation was passing successfully a reading comprehension test. Considering the fact that all participants have passed the test, it would be difficult to assume that the HFA group's lower scores on metaphorical construction in this study was based on reading comprehension. However, according to Giora (2012), weak central coherence cannot explain all findings concerning context effects. For instance, inconsistent with the received view, when familiar and unfamiliar metaphors were compared, it was novelty rather than metaphorically that mattered.

Unlike Gernsbacher and Pripas-Kapit's (2012) conclusion, Whyte, Nelson, and Scherf (2014) found in their study with ASD children that both syntax and ToM abilities predict idiom comprehension success. Basing on their findings, they emphasized that advanced ToM abilities also appear to be important for idiom comprehension in children with ASD because ToM abilities predicted relative deficits in idiom comprehension even after controlling for the contributions of basic language abilities. It has been suggested that both linguistic skills and advanced ToM abilities contribute to idiom comprehension for children with ASD. Similarly, Whyte and Nelson (2015) reported that a combination of basic language skills and ToM abilities may play a role in the development of nonliteral language comprehension.

\section{CONCLUSION}

This study'scontribution totheliteraturehas beenshowninthefollowingaspects: (a)construction of a novel measure of LCC that can be used with children with HFA aged 5-11 years; (b) development of proverbial understanding and metaphorical construction measures adapted for use with children with HFA aged 5-11 years; (c) support of the hypothesis that children with HFA perform lower than TD children on proverbial understanding, metaphorical construction, and local coherence measures; these dimensions tap the ability to understand social situations; (d) showing qualitative differences of performance between children with HFA and TD children across the components of the Proverbial Understanding Test and especially the 
application component; and (e) showing that proverbial understanding is predicted mainly in the HFA group by local coherence and verbal ability.

\section{REFERENCES}

American Psychiatric Association. (2000). Diagnostic and statistical manual of mental disorders (4th ed.). Washington, DC: Author.

Baron-Cohen, S. (1989). The autistic child's theory of mind: A case of specific developmental delay. Journal of Child Psychology and Psychiatry, and Allied Disciplines, 30, 285-297.

Baron-Cohen, S. (1995). Mindblindness. Cambridge, MA: MIT Press.

Bauminger-Zviely, N. (2013). Social and academic abilities in children with high-functioning autism spectrum disorders. New York, NY: Guilford Press.

Bennetto, L., Pennington, B. F., \& Rogers, S. J. (1996). Intact and impaired memory functions in autism. Child Development, 67, 1816-1835.

Bigham, S., Boucher, J., Mayes, A., \& Anns, S. (2010). Assessing recollection and familiarity in autistic spectrum disorders: Methods and findings. Journal of Autism and Developmental Disorders, 40, 878-889.

Boucher, J., \& Bowler, D. M. (2008). Memory in autism. Cambridge, United Kingdom: Cambridge University Press.

Campione, J. C., \& Brown, A. L. (1987). Linking dynamic assessment with school achievement. In C. S. Lidz (Ed.), Dynamic assessment (pp. 82-115). New York, NY: Guilford Press.

Dennis, M., Lazenby, A. L., \& Lockyer, L. (2001). Inferential language in high-function children with autism. Journal of Autism and Developmental Disorders, 31, 47-54.

Eales, M. (1993). Pragmatic impairments in adults with childhood diagnoses of autism or developmental receptive language disorder. Journal of Autism and Developmental Disorders, 23, 593-617.

Fein, D., Dunn, M., Allen, D. A., Aram, D., Hall, R., Morris, R., \& Wilson, B. C. (1996). Neuropsychological and language data. In I. Rapin (Ed.), Preschool children with inadequate communication: Developmental language disorder, autism, low IQ (pp. 123-154). London, United Kingdom: Mac Keith Press.

Feuerstein, R., Rand, Y., \& Hoffman, M. B. (1979). The dynamic assessment of retarded performance: The Learning Potential Assessment Device: Theory, instruments, and techniques. Baltimore, MD: University Park Press.

Fisher, Y. (1975). The Hebrew version for ITPA auditory association subtest (Master's thesis). Tel-Aviv, Israel: Tel-Aviv University.

Frith, U. (1989). Autism: Exploring the enigma. Oxford, United Kingdom: Blackwell.

Frith, U., \& Happé, F. (1994). Autism: beyond "theory of mind." Cognition, 50, 115-132.

Gernsbacher, M. A., \& Pripas-Kapit, S. R. (2012). Who's missing the point? A commentary on claims that autistic persons have a specific deficit in figurative language comprehension. Metaphor and Symbol, 27, 93-105.

Gibbs, R. W., Jr. (2008). The Cambridge handbook of metaphor and thought. Cambridge, United Kingdom: Cambridge University Press.

Gibbs, R. W., Jr., \& Beitel, D. (1995). What proverb understanding reveals about how people think. Psychological Bulletin, 118, 133-154.

Giora, R. (2003). On our mind. Oxford, United Kingom: Oxford University Press.

Giora, R. (2012). Introduction: Different? Not different? Metaphor and Symbol, 27, 1-3.

Giora, R., Gazal, O., Goldstein, I., Fein, O., \& Stringaris, K. (2012). Salience and context: Interpretation of metaphorical and literal language by young adults diagnosed with Asperger's syndrome. Metaphor and Symbol, 27, 22-54.

Gold, R., Faust, M., \& Goldstein, A. (2010). Semantic integration during metaphor comprehension in Asperger syndrome. Brain and Language, 113, 124-134. 
Happé, F. (1993). Communicative competence and theory of mind in autism: A test of relevance theory. Cognition, 48, 101-119.

Happé, F., \& Frith, U. (2006). The weak coherence account: Detail-focused cognitive style in autism spectrum disorders. Journal of Autism and Developmental Disorders, 36, 5-25.

Haywood, H. C. (1995, November). Cognitive early education: Confluence of psychology and education. Paper presented at the Second International Congress on Psychology and Education, Madrid, Spain.

Haywood, H. C., \& Lidz, C. S. (2007). Dynamic assessment in practice: Clinical and educational applications. New York, NY: Cambridge University Press.

Haywood, H. C., \& Tzuriel, D. (Eds.). (1992). Interactive assessment. New York, NY: Springer Publishing.

Haywood, H. C., \& Tzuriel, D. (2002). Applications and challenges in dynamic assessment. Peabody Journal of Education, 77, 38-61.

Heaton, R. K., Chelune, G. J., Talley, J. L., Kay G. G., \& Curtiss G. (1993). Wisconsin Card Sorting Test manual: Revised and extended. Odessa, FL: Psychological Assessment Resources.

Honeck, R. P. (1997). A proverb in mind: The cognitive science of proverbial wit and wisdom. Mahwah, NJ: Lawrence Erlbaum Associates.

Jolliffe, T., \& Baron-Cohen, S. (1999). A test of central coherence theory. Linguistic processing in highfunctioning adults with autism or Asperger syndrome: Is local coherence impaired? Cognition, 71, 149-185.

Jurado, M. B., \& Rosselli, M. (2007). The elusive nature of executive functions: A review of our current understanding. Neuropsychological Review, 17, 213-233.

Kasirer, A., \& Mashal, N. (2014). Verbal creativity in autism: Comprehension and generation of metaphoric language in high-functioning autism spectrum disorder and typical development. Frontiers in Human Neuroscience, 8, .615

Kerbel, D., \& Grunwell, P. (1998). A study of idiom comprehension in children with semantic-pragmatic difficulties. Part II: Between-groups results and discussion. International Journal of Language \& Communication Disorders, 33, 23-44.

Kimchi, R., \& Palmer, S. E. (1982). Form and texture in hierarchically constructed patterns. Journal of Experimental Psychology, 8, 521-535.

Kimhi, Y., Shoam-Kugelmas, D., Agam Ben-Artzi, G., Ben-Moshe, I., \& Bauminger-Zviely, N. (2014). Theory of mind and executive function in preschoolers with typical development versus intellectually able preschoolers with autism spectrum disorder. Journal of Autism and Developmental Disorders, 44, 2341-2354. http://dx.doi.org/10.1007/s10803-014-2104-z

Lakoff, G., \& Turner, M. (1989). More than cool reason: A field guide to poetic metaphor. Chicago, IL: University of Chicago Press.

Le Sourn-Bissaoui, S., Caillies, S., Gierski, F., \& Motte, J. (2011). Ambiguity detection in adolescents with Asperger syndrome: Is central coherence or theory of mind impaired? Research in Autism Spectrum Disorders, 5, 648-656.

Levorato, M. C., \& Cacciari, C. (1992). Children's comprehension and production of idioms: The role of context and familiarity. Journal of Child Language, 19, 415-433.

Lidz, C. S., \& Elliott, J. (Eds.). (2000). Dynamic assessment: Prevailing models and applications. Bingley, United Kingdom: Emerald.

MacKay, G., \& Shaw, A. (2004). A comparative study of figurative language in children with autistic spectrum disorders. Child Language Teaching and Therapy, 20(1), 13-32. http://dx.doi .org/10.1191/0265659004ct261oa

Martin, I., \& McDonald, S. (2003). Weak coherence, no theory of mind, or executive dysfunction? Solving the puzzle of pragmatic language disorders. Brain and Language, 85, 451-466.

Martin, I., \& McDonald, S. (2004). An exploration of causes of non-literal language problems in individuals with Asperger syndrome. Journal of Autism and Developmental Disorders, 34, 311-328.

Mashal, N., \& Kasirer, A. (2011). Thinking maps enhance metaphoric competence in children with autism and learning disabilities. Research in Developmental Disabilities, 32, 2045-2054. 
Melogno, S., Pinto, M. A., \& Levi, G. (2012). Metaphor and metonymy in ASD children: A critical review from a developmental perspective. Research in Autism Spectrum Disorders, 6, 1289-1296.

Mieder, W. (1993). Proverbs are never out of season: Popular wisdom in the modern age. New York, NY: Oxford University Press.

Miller, H. L., Odegard, T. N., \& Allen, G. (2014). Evaluating information processing in autism spectrum disorder: The case for fuzzy trace theory. Developmental Review, 34, 44-76.

Minshew, N. J., \& Goldstein, G. (2001). The pattern of intact and impaired memory functions in autism. Journal of Child Psychology and Psychiatry, and Allied Disciplines, 42, 1095-1101.

Nippold, M. A. (1998). Later language development: The school-age and adolescent years (2nd ed.). Austin, TX: Pro-Ed.

Nippold, M. A., \& Sullivan, M. P. (1987). Verbal and perceptual analogical reasoning and proportional metaphor comprehension in young children. Journal of Speech and Hearing Research, 30, 367-376.

Nippold, M. A., Uhden, L. D., \& Schwarz, I. E. (1997). Proverb explanation through the lifespan: A developmental study of adolescents and adults. Journal of Speech, Language, and Hearing Research, $40,245-253$.

Norbury, C. F. (2004) Factors supporting idiom comprehension in children with communication disorders. Journal of Speech, Language, and Hearing Research, 47, 1179-1193.

Norbury, C. F. (2005a). Barking up the wrong tree? Lexical ambiguity resolution in children with language impairments and autistic spectrum disorders. Journal of Experimental Child Psychology, 90, 142-171.

Norbury, C. F. (2005b). The relationship between theory of mind and metaphor: Evidence from children with language impairment and autistic spectrum disorder. British Journal of Developmental Psychology, 23, 383-399.

Olofson, E. L., Casey, D., Oluyedun, O., Van Herwegen, J., Becerra, A., \& Rundblad, G. (2014). Youth with autism spectrum disorder comprehend lexicalized and novel primary conceptual metaphors. Journal of Autism and Developmental Disorders, 44, 2568-2583.

Owens, G., Granader, Y., Humphrey, A., \& Baron-Cohen, S. (2008). LEGO therapy and the social use of language programme: An evaluation of two social skills interventions for children with high functioning autism and Asperger syndrome. Journal of Autism and Developmental Disorders, 38, 1944-1957.

Ozonoff, S. (1995) Reliability and validity of the Wisconsin Card Sorting Test in studies of autism. Neuropsychology, 9, 491-500.

Ozonoff, S., \& Miller, J. N. (1995). Teaching theory of mind: A new approach to social skills training for individuals with autism. Journal of Autism and Developmental Disorders, 25, 415-433.

Ozonoff, S., Pennington, B. F., \& Rogers, S. J. (1991). Executive function deficits in high-functioning autistic individuals: Relationship to theory of mind. Journal of Child Psychology and Psychiatry, and Allied Disciplines, 32, 1081-1105.

Pellicano, E. (2007). Links between theory of mind and executive function in young children with autism: Clues to developmental primacy. Developmental Psychology, 43, 974-990.

Pellicano, E. (2010). Individual differences in executive function and central coherence predict developmental changes in theory of mind in autism. Developmental Psychology, 46, 530-544.

Perner, J., \& Wimmer, H. (1985). "John thinks that Mary thinks that . . " Attribution of second-order beliefs by 5- to 10- year-old children. Journal of Experimental Child Psychology, 39, 437-471.

Persicke, A., Tarbox, J., Ranick, J., \& St. Clair, M. (2012). Establishing metaphorical reasoning in children with autism. Research in Autism Spectrum Disorders, 6, 913-920.

Peterson, D., \& Bowler, D. M. (2000). Counterfactual reasoning and false belief understanding in children with autism, children with severe learning difficulties and children with typical development. Autism, 4, 391-405.

Piaget, J. (1926). The language and thought of the child. London: Paul, Trench, Trübner. 
Plaisted, K. C. (2001). Reduced generalization in autism: An alternative to weak central coherence. In J. A. Burack, T. Charman, N. Yirmiya, \& P. R. Zelazo (Eds.), The development of autism: Perspectives from theory and research (pp. 149-169). Mahwah, NJ: Lawrence Erlbaum Associates.

Rajendran, G., \& Mitchell, P. (2007). Cognitive theories of autism. Developmental Review, 27, 224-260.

Rapin, I., \& Dunn, M. (2003). Update on the language disorders of individuals on the autistic spectrum. Brain Development, 25, 166-172.

Resing, W. C. M. (1997). Learning potential assessment: The alternative for measuring intelligence? Educational and child Psychology, 14, 68-82.

Robinson, S., Goddard, L., Dritschel, B., Wisley, M., \& Howlin, P. (2009). Executive functions in children with autism spectrum disorders. Brain and Cognition, 71, 362-368.

Rom, A., Morag, L., \& Peleg, S. (2007). MAASE spoken language test: New version. Holon, Israel: Yesod Press.

Rundblad, G., \& Annaz, D. (2010). The atypical development of metaphor and metonomy comprehension in children with autism. Autism, 14, 29-46.

Smith-Seemiller, L., Arffa, S., \& Franzen, M. D. (2001). Use of Wisconsin Card Sorting Test short forms with school-age children. Archives of Clinical Neuropsychology, 16, 489-499.

Sperber, D., \& Wilson, D. (1986). Relevance: Communication and cognition (2nd ed.). Oxford, United Kingdom: Blackwell.

Sternberg, R. J., \& Grigorenko, E. L. (2002). Dynamic testing: The nature and measurement of learning potential. New York, NY: Cambridge University Press.

Tager-Flusberg, H. (1991). Semantic processing in the free recall of autistic children: Further evidence for a cognitive deficit. British Journal of Developmental Psychology, 9, 417-430.

Tavernier-Almada, L. (1999). Prejudice, power, and poverty in Haiti: A study of a nation's culture as seen through its proverbs. Proverbium, 16, 325-350.

Tzuriel, D. (1997). A novel dynamic assessment approach for young children: Major dimensions and current research. Educational and Child Psychology, 14, 83-108.

Tzuriel, D. (2001). Dynamic assessment of young children. New York, NY: Kluwer Academic/Plenum Press.

Tzuriel, D. (2002). Children's conceptual and perceptual analogical modifiability test (CCPAM)—construction analogies version. Ramat-Gan, Israel: Bar-Ilan University.

Tzuriel, D. (2007). Transfer effects of teaching conceptual versus perceptual analogies. Journal of Cognitive Education and Psychology, 6, 194-217.

Tzuriel, D. (2012). Dynamic assessment of learning potential. In M. M. C. Mok, A. Y. P. Lee, \& D. C. H. Lau (Eds.), Assessment reform: Asia Pacific initiatives in assessment to inform learning and teaching (pp. 235-255). New York, NY: Springer Publishing.

Tzuriel, D., \& Flor-Maduel, H. (2010). Prediction of early literacy by analogical thinking modifiability among kindergarten children. Journal of Cognitive Education and Psychology, 9, 207-227.

Tzuriel, D., \& George, T. (2009). Improvement of analogical reasoning and academic achievements by the Analogical Reasoning Program (ARP). Educational and Child Psychology, 29, 71-93.

Tzuriel, D., \& Groman, T. (2013). Children's local/central coherence test (CLCCT). Ramat-Gan, Israel: BarIlan University.

Tzuriel, D., \& Valdman, S., (2009). The development of proverbial understanding as a function of analogical, metaphoric, and verbal abilities. Paper presented at the 12th International Conference of the International Association for Cognitive Education and Psychology, Osnabruck, Germany.

Tzuriel, D., Yosef, L., \& Valdman, S. (2008). Young Children's Metaphoric Ability (YCMA) test. Unpublished manuscript.

Visser, E. M., Berger, H. J. C., Van Schrojenstein Lantman-De Valk, H. M. J., Prins, J. B., \& Teunisse, J. P. (2015). Cognitive shifting and externalising problem behaviour in intellectual disability and autism spectrum disorder. Journal of Intellectual Disability Research, 59, 755-766.

Wang, A. T., Lee, S. S., Sigman, M., \& Dapretto, M. (2006). Neural basis of irony comprehension in children with autism: The role of prosody and context. Brain, 129, 932-943. 
Wechsler, D. (1950). Cognitive, conative and non-intellective intelligence. American Psychologist, 5, 78-83. Wellman, H. M., \& Liu, D. (2004). Scaling of theory-of-mind tasks. Child Development, 75, 523-541.

Whyte, E. M., \& Nelson, K. E. (2015). Trajectories of pragmatic and nonliteral language development in children with autism spectrum disorders. Journal of Communication Disorders, 54, 2-14.

Whyte, E. M., Nelson, K. E., \& Kahn, K. S. (2011). Learning of idiomatic language expressions in a group intervention for children with autism. Autism, 17, 449-464.

Whyte, E. M., Nelson, K. E., \& Scherf, K. S. (2014). Idiom, syntax, and advanced theory of mind abilities in children with autism spectrum disorders. Journal of Speech, Language, and Hearing Research, 57, 120-130.

Williams, D. L., Goldstein, G., \& Minshew, N. J. (2006). The profile of memory function in children with autism. Neuropsychology, 20, 21-29.

Wilson, B. A., Alderman, N., Burgess, P. W., Emslie, H. C., \& Evans, J. J. (1996). The behavioral assessment of the dysexecutive syndrome. Edmunds, United Kingdom: Thames Valley Test.

Wimmer, H., \& Perner, J. (1983). Beliefs about beliefs: Representation and constraining function of wrong beliefs in young children's understanding of deception. Cognition, 13, 103-128.

Worth, S., \& Reynolds, S. (2008). The assessment and identification of language impairment in Asperger's syndrome: A case study. Child Language, Teaching and Therapy, 24, 55-71.

Zelazo, P. D. (2004). The development of conscious control in childhood. Trends in Cognitive Sciences, $8,12-17$.

Zelazo, P. D., Jacques, S., Burack, J. A., \& Frye, D. (2002). The relation between theory of mind and rule use: Evidence from persons with autism-spectrum disorders. Infant and Child Development, 11, 171-195.

Correspondence regarding this article should be directed to David Tzuriel, PhD, Bar Ilan University, School of Education, Geha St., Ramat-Gan, 52900, Israel. E-mail: david.tzuriel@biu.ac.il 